\title{
HACIA UNA MEJOR GESTIÓN DE LOS CONFLICTOS SOCIOAMBIENTALES EN EL PERÚ
}

\author{
Anatolia Hortencia Hinojosa Pérez \\ Universidad Católica San Pablo, Arequipa, Perú
}

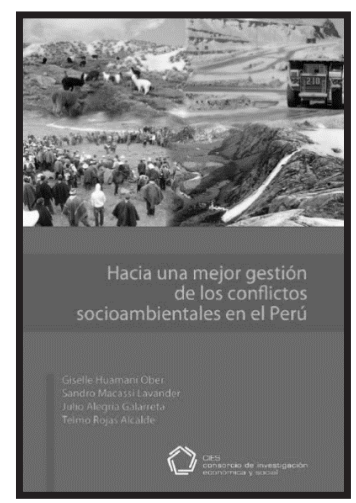

Huamaní, G.; Macasi, S.; Alegría, J. \& Rojas, T. (2012).

"Hacia una mejor gestión de los conflictos socio ambientales en el Perú”.

Lima: Editorial Nova Print S.A.C., 99 págs.

El libro Hacia una mejor gestión de los conflictos socio ambientales en el Perú, ha sido publicado el año 2012 con el apoyo del Consorcio de Investigación Económica y Social (CIES), cuya misión es contribuir al desarrollo del Perú, poniendo en debate nacional problemas clave de nuestra política económica y social. Los autores han publicado este trabajo como resultado de los diagnósticos y las propuestas de mejora identificadas a nivel nacional y regional en el proyecto "Fortalecimiento de capacidades estatales para la prevención y resolución de conflictos socio-ambientales en el Perú".
El objetivo que se proponen ellos, es colaborar con el Estado a mejorar su sistema de gestión de conflictos socio-ambientales con la elaboración de tres diagnósticos: uno a nivel nacional y dos a nivel regional; además de una propuesta de mejora del sistema dirigido a las autoridades del gobierno central, así como a las autoridades regionales que son los llamados a conocer más del tema.

En tal sentido los autores en este trabajo sostienen, que la gestión del conflicto no puede ser manejada como si fuera un área 
temática más dentro de las políticas del Estado. Argumentan que está directamente relacionada con la forma en que la sociedad identifica y se plantea los problemas, los discute, busca soluciones, contiene sus crisis, aprende y construye mejores condiciones para que los conflictos a futuro dejen a la sociedad más fortalecida. En síntesis, sostienen que tiene que ver con los mecanismos, procesos y actitudes para responder a los cambios y solucionar los problemas.

Es de conocimiento público que la conflictividad en nuestro país es transversal en todos los sectores del Estado y todas las regiones, por lo que el trabajo lo enfocan a la conflictividad socio ambiental que es la más grave debido a sus consecuencias sociales, ambientales, políticas, y económicas. Para lograr una reflexión y análisis a casos concretos desarrollaron tres diagnósticos a nivel central, en la región Cajamarca y en la región Cusco donde se desarrolla más la gran y mediana minería. En estos tres diagnósticos produjeron sus correspondientes propuestas y recomendaciones.

Este documento, se ha sistematizado en tres capítulos. Por un lado el primer capítulo lo conforma el diagnóstico de las políticas, procesos, metodologías, protocolos para gestionar los conflictos socio ambientales ordenadas alrededor de siete hipótesis y a partir de los hallazgos encontrados de los diagnósticos a nivel de la región Cajamarca, de la región Cusco y a nivel central. El segundo capítulo contempla el análisis de los elementos que podrían llegar a conformar un Sistema Nacional de Gestión de Conflictos, a partir del mapeo de los actores, los procedimientos, etapas y formas de intervención y los principales procesos para la gestión de conflictos que se ha venido utilizando. El tercer capítulo lo conforma el documento trabajado exclusivamente para desarrollar una perspectiva integral de propuesta y mejoras del Sistema Nacional de Gestión de Conflictos con enfoque de prevención y transformación de los conflictos. Seguramente este trabajo realizado por los autores será un valioso aporte para la solución de los conflictos que se dan con mayor frecuencia en todos los sectores en nuestro país.

Como se mencionó anteriormente, los autores dan a conocer los hallazgos de los diagnósticos según hipótesis, las mismas que se han debatido en los últimos años y tienen que ver con temas de impacto, como el desarrollo de crisis y conflictos alrededor de la minería formal que tuvo una influencia de arrastre en la generación de un sistema de gestión de conflictos. El rol de las mujeres, la perspectiva cultural, negociaciones duras versus negociaciones integrativas, transformación versus manejo de crisis, etc. Lo aquí señalado me lleva a pensar que el Perú es conocido por ser un país extractivo de sus recursos naturales, la normatividad legal no ha ido acorde con el desarrollo de los procesos extractivos para protegernos de los impactos socio ambientales. Así mismo a pesar de que los principios de la mediación, la conciliación y el arbitraje son de aplicación universal y voluntaria a las partes que quisieran utilizarlos, en nuestro país los responsables de la política no utilizan estos medios de resolución de conflictos, por lo que con el aumento de la actividad minera en el país los conflictos se han incrementado. Si tenemos en cuenta la 
existencia de más de 200 conflictos socio ambientales vigentes en nuestro país y que muchos de estos conflictos devienen en desastres políticos como el conflicto de la población de Islay, en Arequipa, contra el proyecto minero "Tía María" de la empresa Southern Copper, podremos notar el alto nivel de importancia del tema y las situaciones de violencia alrededor de operaciones mineras a gran escala.

Por otro lado los autores observaron que la normatividad existente para la resolución de conflictos en las regiones de Cajamarca y Cusco son poco claras y difusas, así mismo señalan que en las entrevistas no se reconocieron los aportes de la sociedad civil en la conducción de los procesos de manejo de conflictos. Por otro lado, los actores en los conflictos se enfocan en sus intereses y en sus posiciones políticas y que los procesos de gestión de conflictos no incorporan las necesidades e intereses de los grupos más excluidos; lo que nos lleva a reflexionar que todo esto sucede debido a la falta de articulación entre los niveles locales y regionales sobre las problemáticas en torno a los recursos naturales y cómo responder a ellas, lo que recrudece aún más, cuando se necesita articular con el nivel nacional. Por otro lado la falta de articulación entre los planes de desarrollo local concertado y las actividades de las empresas mineras, que en muchos casos, son orientadores de la inversión privada, son desconocidos no solo por la empresa, sino también por las comunidades, quienes no han sido consultadas. En consecuencia, se han visto excluidas del proceso de toma de decisiones, lo que agudiza la situación de conflicto.
En el trabajo responden a la pregunta ¿Qué es el Sistema Nacional de Gestión de Conflictos? Se define un sistema de gestión de conflictos como un conjunto de instituciones, principios, normas, procedimientos, técnicas e instrumentos que conforman una estructura mediante la cual el Estado asegura la gestión integrada, participativa y multisectorial de los conflictos. Sin embargo, una definición más extensa también incluye procesos, el recurso humano, el entorno físico, los mecanismos de control, así como los atributos menos visibles, como las actitudes, creencias y valores de los miembros de la organización. Además, un sistema incluye relaciones e interacción de todo el sistema con las partes y de estas con el todo. Sin embargo los autores advierten que en las regiones, los impactos de este sistema son débiles. Observan que la insuficiente normatividad hace que las intervenciones institucionales no estén compatibilizadas ni jerarquizadas, lo que provoca que el accionar del MINEM, INGEMMET, MINAM, ANA, el Gobierno Regional, los Gobiernos provinciales y locales se contrapongan y no armonicen entre sí. La definición que los autores dan a un sistema de gestión de conflictos, es un aporte fundamental, una conceptualización que todos los actores deberían conocer y aplicar para la solución de los conflictos en nuestro país.

Finalmente en el trabajo presentan una propuesta y mejoras del Sistema Nacional de Gestión de Conflictos, el objetivo central de la iniciativa impulsada por el CIES ha sido diagnosticar y desarrollar un conjunto de mejoras al Sistema Nacional de Gestión de Conflictos. Esta propuesta la han organizado en dos niveles: Propuestas a nivel Nacional y 
Propuestas a nivel Regional, en cada uno de estos niveles han desarrollado cinco líneas de acción claves, para construir un sistema coherente, integrado y articulado para la gestión de conflictos: Institucionalidad, Capacidades, Mecanismos y condiciones, Sociedad civil, y Comunicación. Recomiendan, que las propuestas de mejora implican desarrollos institucionales a corto, mediano y largo plazo, pero que van a depender de la voluntad política realmente existente para que una propuesta de esta naturaleza se implemente en su totalidad. Sin embargo, el desafío de los autores es mostrar y discutir la política pública sobre gestión de conflictos, con un documento que proponga un horizonte diferente a como a la fecha se ha actuado.

A través de un lenguaje sencillo y claro, los autores en el trabajo que nos presentan, dan a conocer que el Perú no cuenta con elementos integrados de un sistema para la gestión de conflictos. Sin embargo, nos dan la esperanza de que existen elementos de una institucionalidad con el potencial de articularse y desarrollar un sistema de gestión de conflictos en el futuro. Así mismo plantean la necesidad de ampliar el rol y capacidades de los actores regionales del desarrollo para intervenir con soluciones dialogadas en los conflictos, capacitando a instituciones y líderes de la sociedad civil, que contribuyan al encauzamiento de los conflictos. Acotan también la urgencia de fortalecer procesos de comunicación y transparencia para una participación informada de las comunidades y de la sociedad a nivel regional, también sugieren el desarrollo de talleres de periodismo preventivo, a fin de incorporar en la práctica periodística los principios de la prevención y transformación de conflictos e involucrar a los medios de comunicación en procesos de diálogo y seguimiento del cumplimiento de los acuerdos. Tal vez hubiera sido enriquecedor que los autores estudiaran también los conflictos en la región Arequipa, dado que esta región cuenta con dos yacimientos mineros importantes, como es el caso de Cerro Verde en plena actividad y el proyecto Tía María que se viene retrasando precisamente por este problema.

El libro es importante porque permite conocer y entender la problemática que aborda, dado que corresponde a procesos complejos; además los autores involucran a sus lectores para que sean partícipes en la solución de estos conflictos como actores proactivos desde los diferentes roles que desempeñan en nuestra sociedad. De esta manera contribuyen con el objetivo de colaborar con el Estado a mejorar su sistema de gestión de conflictos socio-ambientales. 